\title{
Supporting Information \\ Oxygen Evolution and Reduction on Two-Dimensional Transition \\ Metal Dichalcogenides
}

\author{
Naiwrit Karmodak, ${ }^{*}$ Luca Bursi, and Oliviero Andreussi* \\ Department of Physics, University of North Texas, Denton, TX 76203, USA \\ E-mail: naiwrit.karmodak@unt.edu; oliviero.andreussi@unt.edu
}

\section{S-I. Computational Details}

The calculations are performed using the density functional theory employing the planewaves DFT code Quantum Espresso. ${ }^{1}$ To incorporate the the electron exchange interactions, the vdW-DF2-C09 van der Waals (vdW) density functional ${ }^{2-4}$ is used and the the electron ion interactions are treated using the library of SSSP efficiency pseudopotentials. ${ }^{5,6}$ Wavefunction and charge density cutoffs of 45 and 360 Ry respectively are chosen. The Brillouin Zone is sampled using a $\Gamma$-centered Monkhorst-Pack grid, with a maximum k-point distance along the periodic distance less than $0.1 \AA^{-1}$. The binding energies of the $2 \mathrm{D}$ structures and the adsorption energies of the intermediates have been calculated using a Marzari-Vanderbilt cold smearing of $0.02 \mathrm{Ry}^{7}$ Along the non-periodic direction, a vacuum space of $20 \AA$ is added to remove fictitious interactions between the periodic images. Furthermore, the parabolic correction scheme as derived by Andreussi and Marzari ${ }^{8}$ for vacuum or in continuum dielectric medium simulations and extended in Ref. ${ }^{9}$ for continuum electrolyte media is used to introduce corrections for electrostatic interactions along the direction perpendicular to the slab. 
The electrochemical interface is defined by an implicit solvation model. In this model, the $2 \mathrm{D}$ material surfaces and the adsorbed intermediates such as $\mathrm{OH}^{*}, \mathrm{O}^{*}$ and $\mathrm{OOH}^{*}$ ) are treated quantum mechanically following the symmetric slab approach. The aqueous medium is defined by a polarizable continuum (PC) with dielectric permittivity equivalent to 78.3. The dielectic cavity geometry is determined from the electronic density of the quantum mechanical system following the self-consistent continuum solvation (SCCS) model ${ }^{8,10,11}$ implemented in the ENVIRON-1.1 module ${ }^{12}$ and coupled with the Quantum Espresso code. ${ }^{13}$ The absolute reference for electrostatic potentials is represented by the electrostatic potential within the bulk of the dielectric continuum. By varying the total number of the electrons in the system, the potential of the electrode is changed, where the net charges are compensated by the counter charges of the electrolyte ions in the electrochemical double layer within the solution. Charge neutrality insures that the reference potential in the bulk of the solution is flat. From the Fermi energy of the system, the applied electrode potential is computed. This potential as obtained in the absolute electrostatic potential scale. The screening of the electrolyte double layer is described by the Gouy-Chapman-Stern model, where the electrolyte counter-charges are defined using the Gaussian-shaped counter-charge layers (width: $0.5 \AA$ ), placed on both sides of the 2D material surface at a distance of $5 \AA$ from the topmost atom along the non-periodic z-axis. ${ }^{9,14}$ The electrosorption free energies of the adsorbates are calculated using Grand-Potential Simulation scheme ${ }^{15}$ as discussed in the SI of ref. ${ }^{16}$ The desorption barrier of the aqueous decomposition products in the fourth screening step are performed using the nugged elastic band (NEB) calculations, ${ }^{17,18}$ where the solvent interactions on the transition state geometries are considered following the continuum solvation approach. 


\section{S-II Thermodynamic and Dynamic stability of the TMDC materials}

Thermodynamic Stability: In the initial screening step, the thermodynamic stability of the materials from the $\mathrm{C} 2 \mathrm{DB}$ and Materials Cloud databases are determined based upon the thermodynamic data reported in these databases. For analysing the thermodynamic stability of the materials from C2DB database, the convex hull energies denoted as $E_{\text {hull }}$ values are used. The $E_{\text {hull }}$ is defined as the formation energy of the most stable phase with the similar chemical composition as the 2D material. For example the heat of formation energy per atom for a compound with the chemical formula $A_{x} B_{y}$ is defined by:

$$
E_{\text {hull }}=\left[E\left(A_{x} B_{y}\right)-x * E(A)-y * E(B)\right] /(x+y)
$$

Here, $E\left(A_{x} B_{y}\right.$ correspond to the total energy of the $2 \mathrm{D}$ materials. $\mathrm{E}(\mathrm{A})$ and $\mathrm{E}(\mathrm{B})$ are the total energies of the elements $\mathrm{A}$ and $\mathrm{B}$ in the standard states. The $2 \mathrm{D}$ material with a heat of formation energy less than $E_{\text {hull }}+0.2 \mathrm{eV} /$ atom are denoted as thermodynamically stable in C2DB database. Among the initial set of the 2D TMDC monolayers from C2DB database, we have screened off all the materials for which the formation energy values are reported to be lower than this thermodynamic stability threshold value.

On the other hand, in the Materials Cloud database, the exfoliation energies from the corresponding 3D van-der-Waals bulk phases have been used to analyse the thermodynamic stability of the 2D materials. The easily exfoliable materials with exfoliation energies smaller than $35 \mathrm{meV} / \AA^{2}$ have been considered as the thermodynamically most stable materials. Therefore, the TMDC monolayers from Materials Cloud database with the exfoliation energies greater than $35 \mathrm{meV} / \AA^{2}$ have been removed from the initial dataset.

While the $E_{\text {hull }}$ values reported in $\mathrm{C} 2 \mathrm{DB}$ database are calculated with a different set of computational parameters and DFT xc-functional, however, comparing the $E_{\text {hull }}$ values for a 
representative set of 35 compounds with the exfoliation energies reported in Materials Cloud database the authors estimated the errors to be less than $0.1 \mathrm{eV} /$ atom. $^{19}$

Dynamic Stability: The dynamic stability of the compounds have been determined based upon the phonon frequencies reported in ref. ${ }^{19,20}$ The respective phonon frequencies could be visualized for the TMDC monolayers from the web-link cmrdb.fysik.dtu.dk/c2db/ and www.materialscloud.org/discover/2dstructures/ Positive hessian eigenvalues corresponds to the kinetic stability of the materials. However, some of the synthetically known 2D materials, for example $1 \mathrm{~T}-\mathrm{MoS}_{2}, 1 \mathrm{~T}-\mathrm{VTe}_{2}$ and $1 \mathrm{~T}-\mathrm{TiS}_{2}$, show theoretical hessian eigenvalues lower than $-2 \mathrm{eV} / \AA^{2} \cdot{ }^{21-23}$ Therefore, we have used a more relaxed criterion to determine the dynamic stability of the materials. We have considered the materials with minimum hessian eigenvalues greater than $-2 \mathrm{eV} / \AA^{2}$ as dynamically stable and suitable for calculating the catalytic properties in the proceeding steps. 


\section{S-III Selection criteria for the kinetic barrier threshold $\left(\Delta E_{k i n}\right)$ in the step 4 of screening workflow}

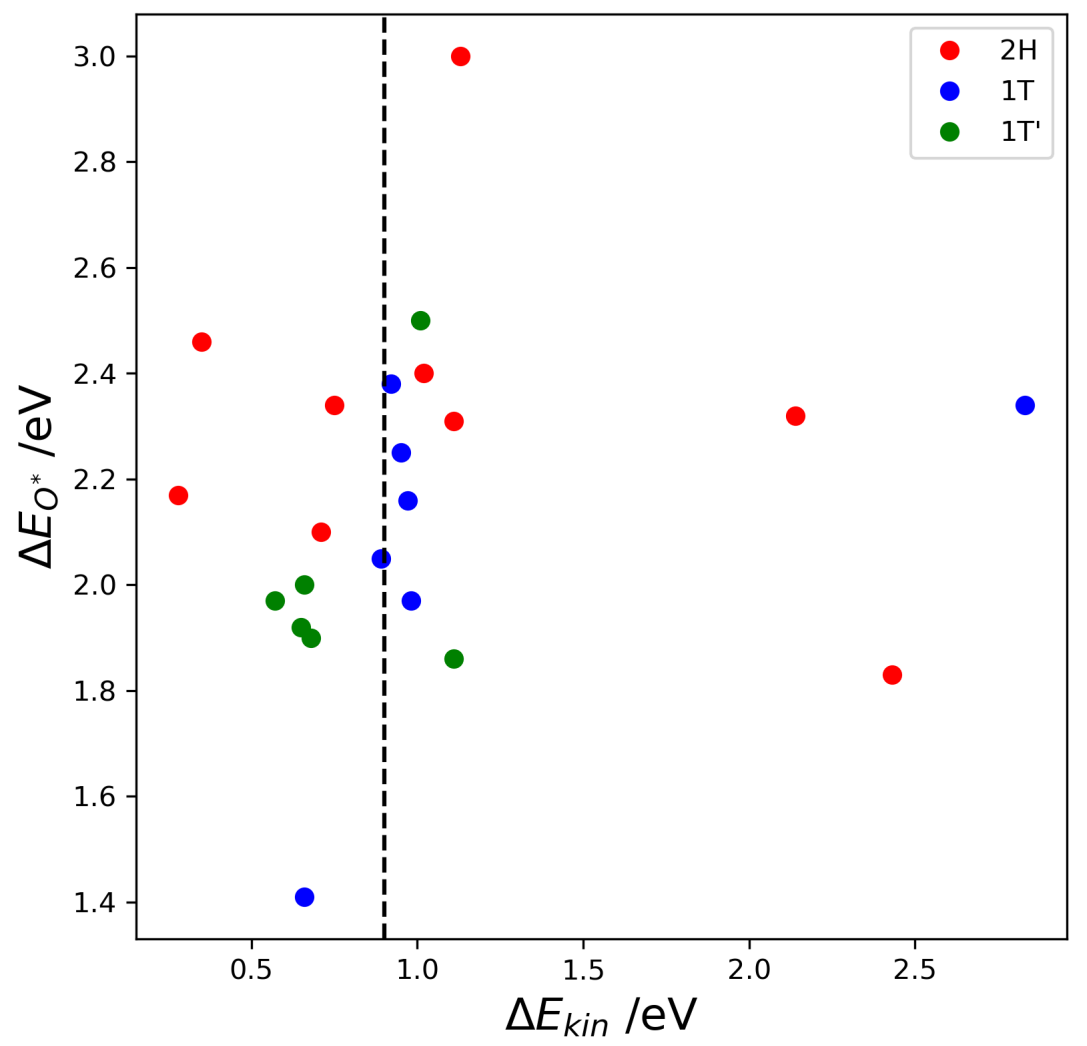

Figure S1: The kinetic barrier for the desorption of the aqueous decomposition products $\left(\Delta E_{k i n}\right)$ as per the reaction pathway shown in Figure 3a (main text) is plotted with respect surface adsorption energy of $\mathrm{O}^{*}$ intermediates $\left(\Delta E_{\mathrm{O}^{*}}\right)$. The color dots represent the different TMDC phases as indicated in the inset of the figure. The black vertical dotted line at $\Delta E_{k i n}$ $=0.9 \mathrm{eV}$ shows the selection threshold for $\Delta E_{k i n}$. The materials with $\Delta E_{k i n}$ greater than this value are considered as kinetically stable towards decompostion to the aqueous products. 


\section{S-IV Comparison of Overpotentials values calculated using grand canonical simulations and CHE method}

The reaction free energy values $\Delta G_{1}, \Delta G_{2}, \Delta G_{3}$ and $\Delta G_{4}$ for the four proton coupled electron transfer step as shown in reaction scheme 2a for OER are determined by the eq. S19 to eq. S5:

$$
\begin{gathered}
*+\mathrm{H}_{2} \mathrm{O} \rightarrow \mathrm{OH}^{*}+\mathrm{H}^{+}+e^{-}, \Delta G_{1}=\Delta G_{O H^{*}} \\
\mathrm{OH}^{*} \rightarrow \mathrm{O}^{*}+\mathrm{H}^{+}+e^{-}, \Delta G_{2}=\Delta G_{O^{*}}-\Delta G_{O H^{*}} \\
\mathrm{O}^{*}+\mathrm{H}_{2} \mathrm{O} \rightarrow \mathrm{OOH}^{*}+\mathrm{H}^{+}+e^{-}, \Delta G_{3}=\Delta G_{O O H^{*}}-\Delta G_{O^{*}} \\
\mathrm{OOH}^{*} \rightarrow \mathrm{O}_{2}+*+\mathrm{H}^{+}+e^{-}, \Delta G_{4}=\Delta G_{\mathrm{O}_{2}}-\Delta G_{O O H^{*}}
\end{gathered}
$$

On the other hand, for ORR the corresponding free energy values are obtained from

$$
\begin{gathered}
*+\mathrm{O}_{2}+\mathrm{H}^{+}+e^{-} \rightarrow \mathrm{OOH}^{*}, \Delta G_{1}=\Delta G_{O O H^{*}}-\Delta G_{\mathrm{O}_{2}} \\
\mathrm{OOH}^{*}+\mathrm{H}^{+}+e^{-} \rightarrow \mathrm{O}^{*}+\mathrm{H}_{2} \mathrm{O}, \Delta G_{2}=\Delta G_{O^{*}}-\Delta G_{O O H^{*}} \\
\mathrm{O}^{*}+\mathrm{H}^{+}+e^{-} \rightarrow \mathrm{OH}^{*}, \Delta G_{3}=\Delta G_{O H^{*}}-\Delta G_{O^{*}} \\
\mathrm{OH}^{*}+\mathrm{H}^{+}+e^{-} \rightarrow \mathrm{H}_{2} \mathrm{O}+*, \Delta G_{4}=-\Delta G_{O H^{*}}
\end{gathered}
$$

These equations are obtained from the formation free energies of the different intermediates $\Delta G_{O H} *, \Delta G_{O} *$ and $\Delta G_{O O H} *$ as given in the following reactions, Eqs. S10 - S12.

$$
\begin{aligned}
& *+\mathrm{H}_{2} \mathrm{O} \stackrel{\Delta G_{O H *}}{\longrightarrow} \mathrm{OH}^{*}+\mathrm{H}^{+}+e^{-} \\
& *+\mathrm{H}_{2} \mathrm{O} \stackrel{\Delta G_{O *}}{\longrightarrow} \mathrm{O}^{*}+2 \mathrm{H}^{+}+2 e^{-}
\end{aligned}
$$




$$
*+2 \mathrm{H}_{2} \mathrm{O} \stackrel{\Delta G_{O O H *}}{\longrightarrow} \mathrm{OOH}^{*}+3 \mathrm{H}^{+}+3 e^{-}
$$

In the grand canonical simulations, the $\Delta G_{O H} *, \Delta G_{O} *$ and $\Delta G_{O O H} *$ are calculated from the grand canonical free energies of $\mathrm{OH}^{*}, \mathrm{O}^{*}$ and $\mathrm{OOH}^{*}$ intermediates following eq. S13

$$
\Delta G_{\mathrm{XH}^{*}}^{G C}(\Phi)=J_{\mathrm{XH}^{*}}(\Phi)-J_{*}(\Phi)+\Delta E_{Z P E}-T \Delta S_{a d s}
$$

where $\mathrm{XH}^{*}$ denotes the intermediates $\mathrm{OH}^{*}, \mathrm{O}^{*}$ and $\mathrm{OOH}^{*} \cdot J_{\mathrm{XH}^{*}}(\Phi)$ and $J_{*}(\Phi)$ are the grand canonical free energy of the 2D material surface with and without the adsorbed intermediates respectively, while $\Delta E_{Z P E}$ and $T \Delta S_{a d s}$ are the zero-point energy and entropic corrections. The grand canonical overpotential $\eta_{G C}$ corresponds to the maximum/minumum potential for OER/ORR at which all the reaction intermediate steps (eq. S19 to eq. S5 and eq. S6 to eq. S9 respectively for OER and ORR) becomes downhill in energy.

In the CHE method, the reaction free energies are calculated following the eq. S14

$$
\Delta G_{\mathrm{XH}^{*}}^{C H E}(\Phi)=E_{\mathrm{XH}^{*}}^{D F T}-E_{*}^{D F T}-n * E_{\mathrm{H}_{2} \mathrm{O}}^{D F T}+\frac{n}{2} * E_{\mathrm{H}_{2}}^{D F T}+\Delta E_{Z P E}-T \Delta S_{a d s}-n * e \phi
$$

Here, $\mathrm{n}$ denotes the number of electrons. The $E_{\mathrm{XH}^{*}}^{D F T}$ and $E_{*}^{D F T}$ are the DFT calculated energy of the $2 \mathrm{D}$ mataerial surface with and without the intermediates. $E_{\mathrm{H}_{2} \mathrm{O}}^{D F T}$ and $E_{\mathrm{H}_{2}}^{D F T}$ are the gas phase energies of $\mathrm{H}_{2} \mathrm{O}$ and $\mathrm{H}_{2}$.

The overpotential $\eta_{C H E}$ is defined by the maximum potential needed to downhill all the intermediate reaction steps. The overpotential value $\left(\eta_{C H E}\right)$ at standard condition is defined by eq. and eq. S16 respectively for OER and ORR.

$$
\eta_{C H E}^{O E R}=\frac{\Delta G_{p d s}}{e}-1.23 \mathrm{~V}
$$




$$
\eta_{C H E}^{O R R}=\frac{\Delta G_{p d s}}{e}+1.23 \mathrm{~V}
$$

The $\Delta G_{p d s}$ corresponds to the free energy for the potential determining step and e is the electronic charge. $1.23 \mathrm{~V}$ is the oxidation potential required for the ideal catalyst surface for the oxidation of $\mathrm{H}_{2} \mathrm{O}$ to $\mathrm{O}_{2}$ or at standard electrochemical conditions in OER or vice-versa in ORR. The $\Delta G_{p d s}$ is free energy of the potential determining step and obtained using the eq. S17

$$
\Delta G_{p d s}=\max \left[\Delta G_{1}, \Delta G_{2}, \Delta G_{3}, \Delta G_{4}\right]
$$

In Figure S2 the $\eta_{G C}$ and $\eta_{C H E}$ values are plotted for the best materials obtained after the first three steps of the screening workflow. The materials are arranged in the increasing order as per the overpotential values obtained from grand canonical calculations. For most of the materials, the CHE calculations show slightly higher overpotentials values compared to the $\eta_{G C}$ values. 

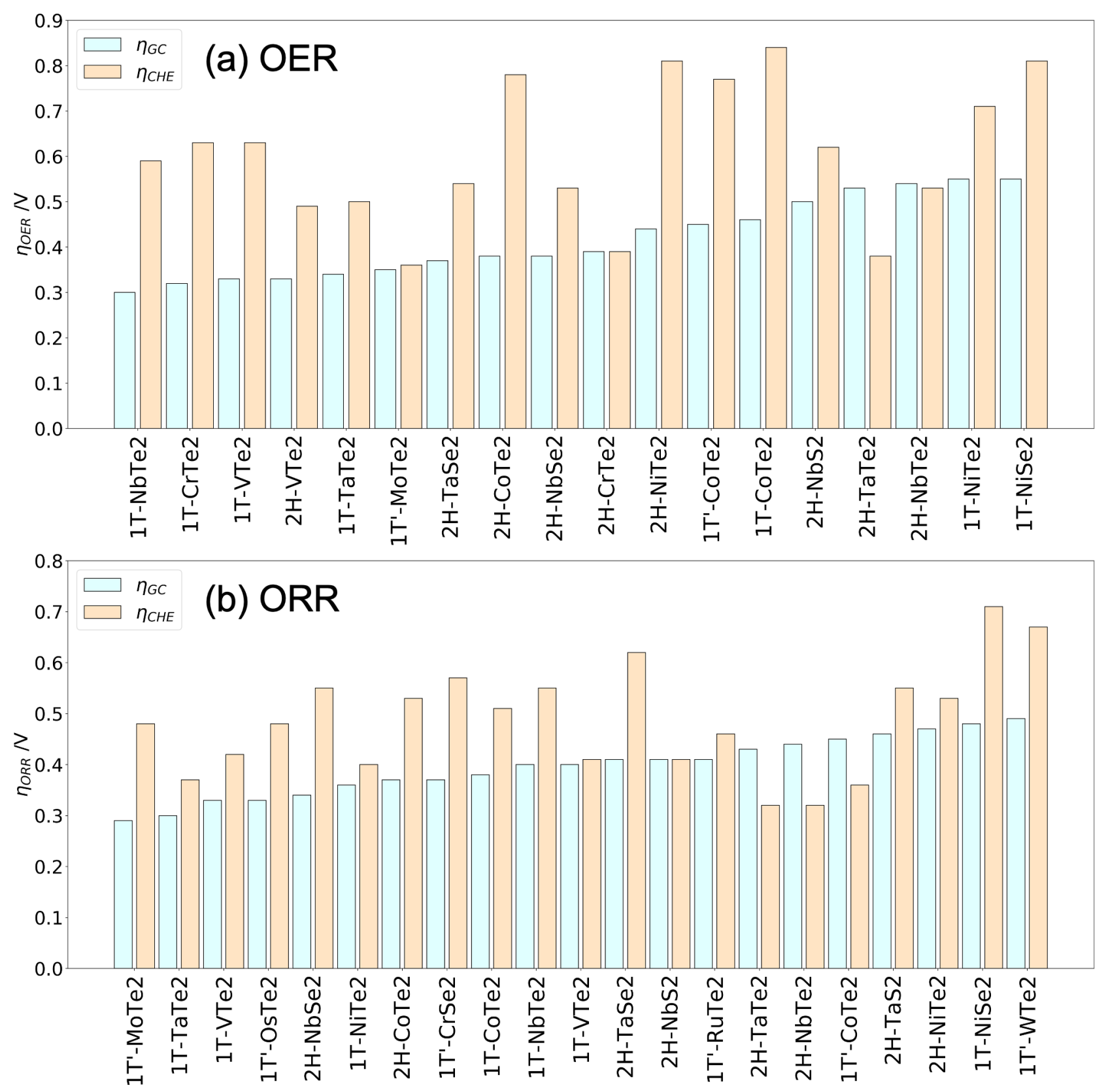

Figure S2: A comparison of the overpotential values for OER (a) and ORR (b) calculated using the Grand Canonical simulations $\left({ }_{G C}\right)$ and CHE $\left(\eta_{C H E}\right)$ method for the TMDC monolayers screened after the first three cycles of the screening workflow. The materials are ordered as per the increasing values of the overpotentials obtained from Grand Canonical simulations. The cyan bar denotes the ${ }_{G C}$ values and bisque colored bars represent the ${ }_{C H E}$ values. The chemical formula for the materials are denoted below the each plot. 


\section{S-V The Decomposition pathway for the surface oxidized}

\section{TMDCs}

At the oxidizing potentials for the screened 2D materials, the formation of multiple $\mathrm{O}^{*}$ intermediates on a single surface site is found to be more preferable than adsorption on different sites. With this structural arrangement of $\mathrm{O}^{*}$ intermediates, the probable decomposition pathway corresponds ${ }^{21-23}$ to the desorption of the surface chalcogen atom with one or both of the attached $\mathrm{O}$ atoms. Therefore, $\mathrm{XO}_{2}$ or $\mathrm{XO}$ species could be the decomposition products as shown in Figure S3.

The initial steps denoted by the orange arrows leading to the adsorption of $\mathrm{O}^{*}$ intermediates on the basal plane (steps (a) to (c) in Figure S3) would be electrochemical process, whereas the desorption of aqueous products (step (c) to (d) or (e)), either $\mathrm{XO}_{2}$ or $\mathrm{XO}$, would be thermally controlled. At the applied overpotential, the thermodynamic barrier for the initial two steps (formation of $\mathrm{O}^{*}$ intermediates on the basal surface) would be thermodynamically favored process. In this situation, the thermal desorption of $\mathrm{XO}_{2}$ or $\mathrm{XO}$ ion will form the reaction-limiting step, determining the overall propensity towards decomposition. To fully characterize these processes, we calculate both the thermodynamic free energies and kinetic barrier of the thermal desorption pathways. The materials that are thermodynami-

cally more stable than the decomposition products at operating conditions are identified as promising catalysts. In addition, to account for the possibility of metastable materials, in which the decomposition process is significantly unlikely at standard conditions, compounds with a large kinetic barrier for decomposition are also selected as potential catalysts. 


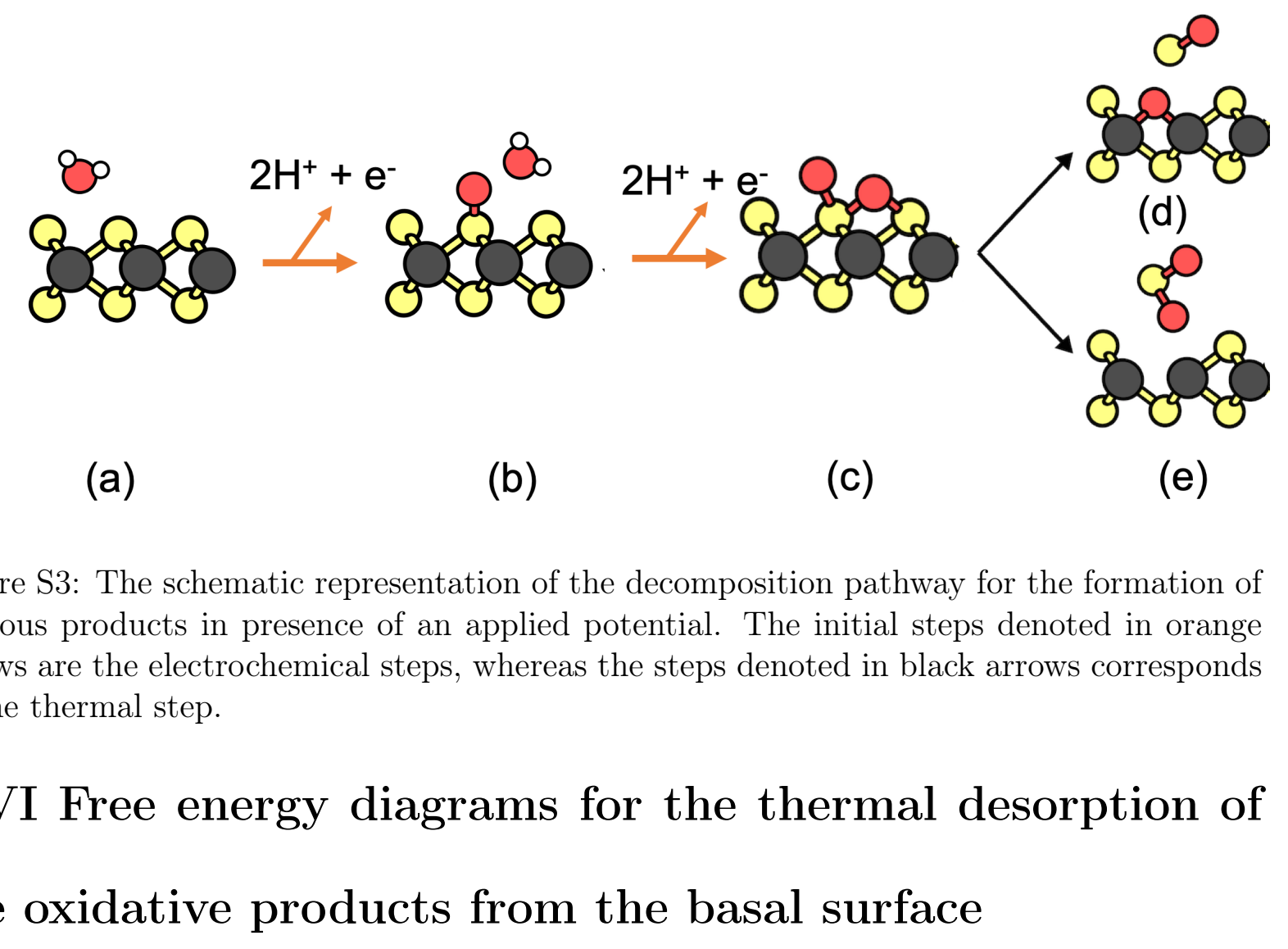

The Free energy diagram and the atomic arrangements of the initial, final and the transition state structures for the nine experimentally known TMDC monolayers with the best electrocatalytic activities identified after the four screening steps are shown in Figure S3, S4 and S5. In the free energy diagram, the transition states are denoted in red solid lines, whereas the initial states are denoted in black. The intermediate states and the final states are represented in blue solid lines. The corresponding energies values are given in $\mathrm{eV}$. 

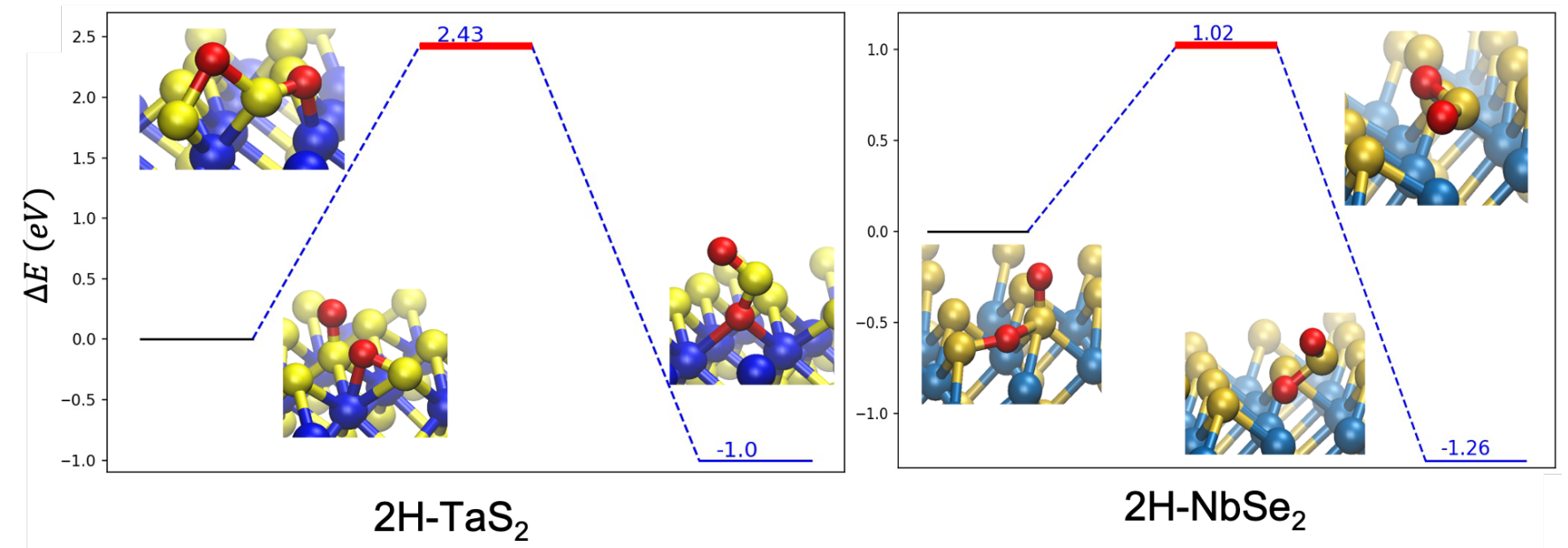

Figure S4: The desorption free energy diagram for 2H-phase. Only two TMDC monolayers, $\mathrm{TaS}_{2}$ and $\mathrm{NbSe}_{2}$ with $2 \mathrm{H}$ phase are found to satisfy all the criteria in the four screening steps. The most preferred desorption product in both these monolayers is $\mathrm{XO}_{2}$ species. 


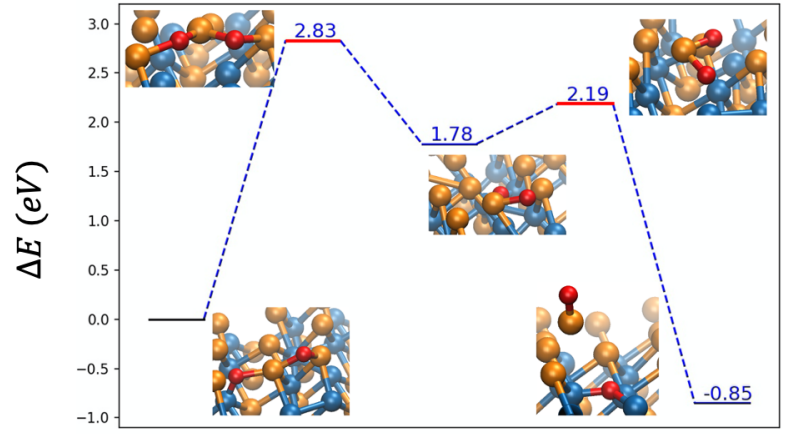

$1 \mathrm{~T}-\mathrm{NbTe}_{2}$

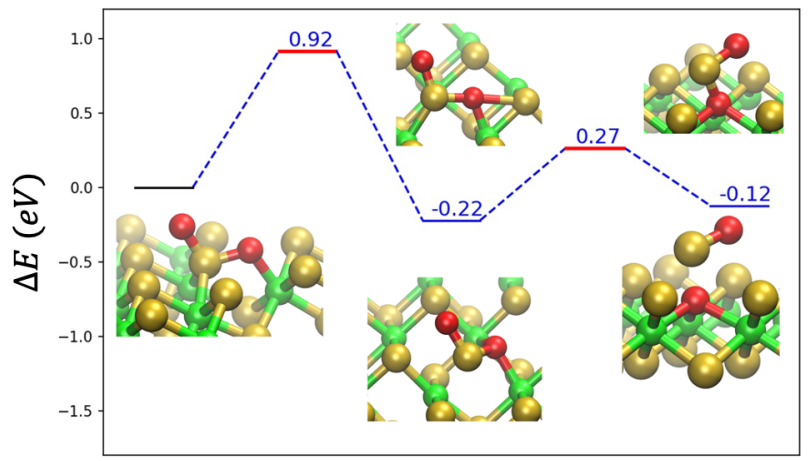

$1 \mathrm{~T}-\mathrm{NiSe}_{2}$

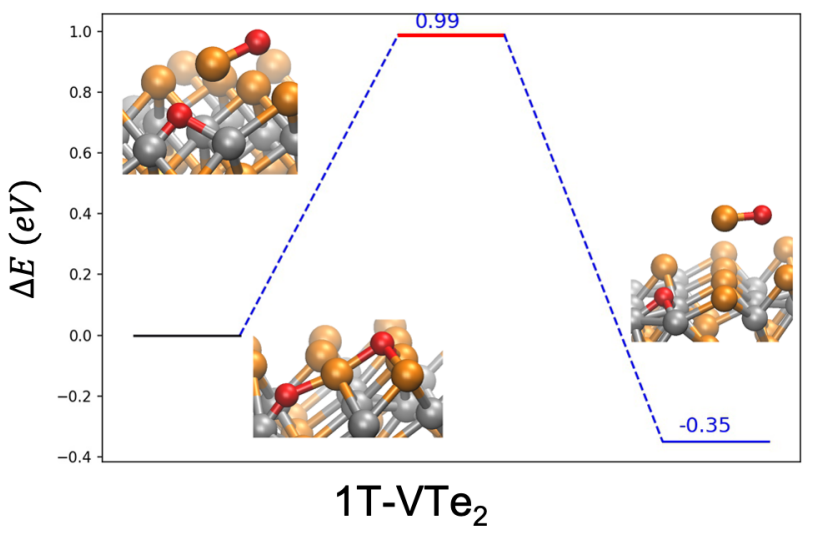

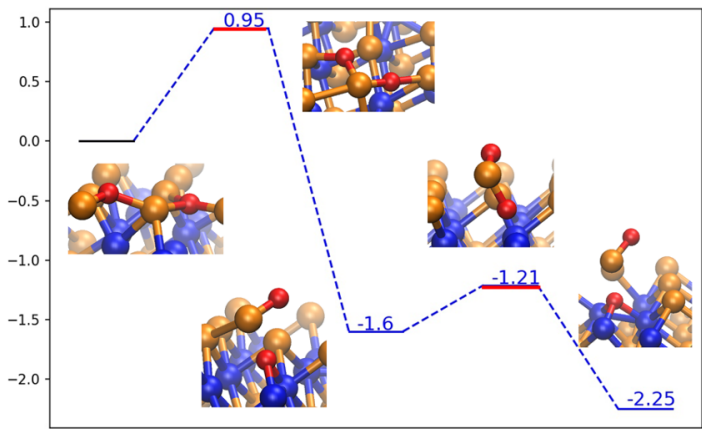

$1 \mathrm{~T}-\mathrm{TaTe}_{2}$

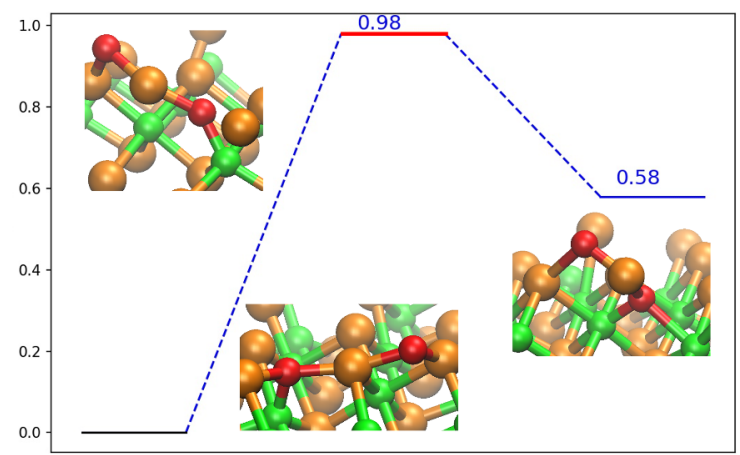

$1 \mathrm{~T}-\mathrm{NiTe}_{2}$

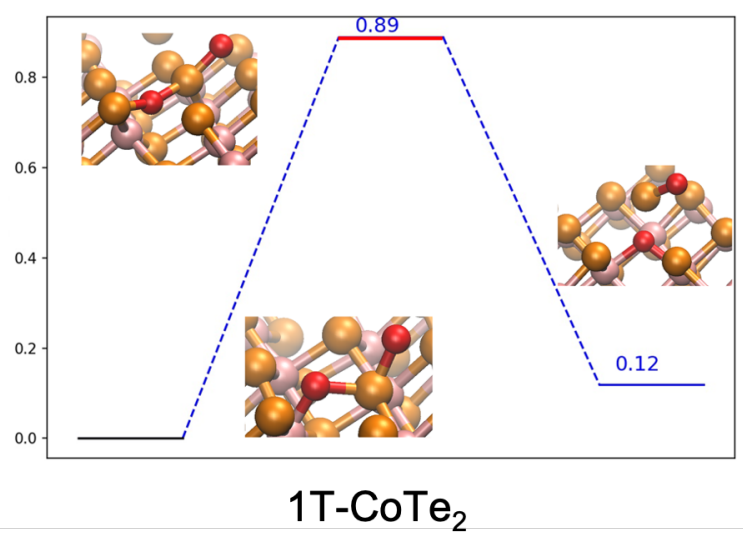

Figure S5: The desorption free energy diagram for 1T-phase monolayers. With 1T-phase, we have identified six experimentally known monolayers with higher electrocatalytic activity for OER/ORR. For the four monolayers with the molecular formula $\mathrm{NbTe}_{2}, \mathrm{TaTe}_{2}, \mathrm{VTe}_{2}$, and $\mathrm{NiSe}_{2}$, the formation of $\mathrm{XO}$ species corresponds to the most preferable aqueous product. In the other two monolayers, $\mathrm{NiTe}_{2}$ and $\mathrm{CoTe}_{2}$, the preferred aqueous decomposition product corresponds to $\mathrm{XO}_{2}$ species. 


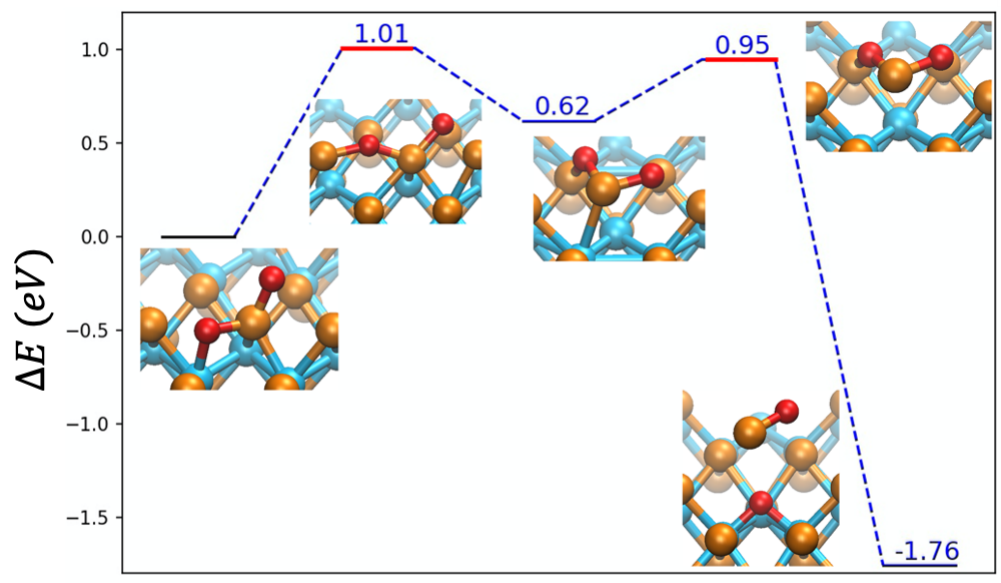

$1 \mathrm{~T}^{\prime}-\mathrm{MoTe}_{2}$

Figure S6: The desorption free energy diagram for $1 \mathrm{~T}^{\prime}-\mathrm{MoTe}_{2}$. Following the formation of $\mathrm{O}^{*}$ intermediate, the major aqueous product corresponds to TeO species. The desorption process is found to proceed via two transition states and an intermediate state. 


\section{S-VII Surface Pourbaix stability of the most active mate-}

\section{rials}

The electrosorption free energy calculations with the grand potential simulation scheme would lead to erroneous effect on the surface energy calculations with higher adsorbate coverage due to the formation of inter-adsobate complexes. Therefore, we used CHE scheme to determine the surface Pourbaix diagrams as shown in Figures S7 to S9. Based upon the adsorption of the possible oxygenated species, the free energies denoted as $\Delta G_{p b x}$ are calculated considering the following reaction pathway:

$$
*+n \mathrm{H}_{2} \mathrm{O} \rightleftharpoons \mathrm{H}_{\mathrm{n}} \mathrm{O}_{\mathrm{m}}^{*}+(2 m-n)\left(\mathrm{H}^{+}+e^{-}\right)
$$

Here, ${ }^{*}$ and $\mathrm{H}_{\mathrm{n}} \mathrm{O}_{\mathrm{m}}^{*}$ denote the bare and oxygenated surfaces. The free energy values $\Delta G_{p b x}$ are calculated based on the methods described by ref () and the entropic and zero point energy corrections according to the following equation

$$
\Delta G_{p b x}=G_{\mathrm{H}_{\mathrm{n}} \mathrm{O}_{\mathrm{m}}^{*}}-G_{*}-m G_{\mathrm{H}_{2} \mathrm{O}}+(2 m-n) *\left(\frac{1}{2} G_{\mathrm{H}_{2}}-U_{R H E}\right)
$$

$\mathrm{m}$ and $\mathrm{n}$ refer to the number of oxygen and hydrogen atoms in the adsobed oxygenated species. For some of the materials ( all T'-TMDCs, 2H-NbS $2, \mathrm{H}-\mathrm{NbSe} 2,2 \mathrm{H}-\mathrm{TaS} 2,2 \mathrm{H}-$ $\mathrm{TaSe}_{2}$ and $1 \mathrm{~T}-\mathrm{MoTe}_{2}$ ), we have observed that beyond the $50 \%$ coverage, $\mathrm{OH}^{*}$ and $\mathrm{OOH}^{*}$ intermediates show weak binding affinity and desorb away from the surface during relaxation. The Surface Pourbaix diagram for these materials are plotted considering up to 50\% surface coverage with the intermediates. The other materials are found to favourably allow full surface coverage of the intermediates. In the Surface Pourbaix plots, the red and blue regions denote the observed potential range within which these materials are found to show higher activity for ORR and OER respectively. These potential ranges are obtained based upon the electrosorption free energy calculations in the third step of the screening workflow. 

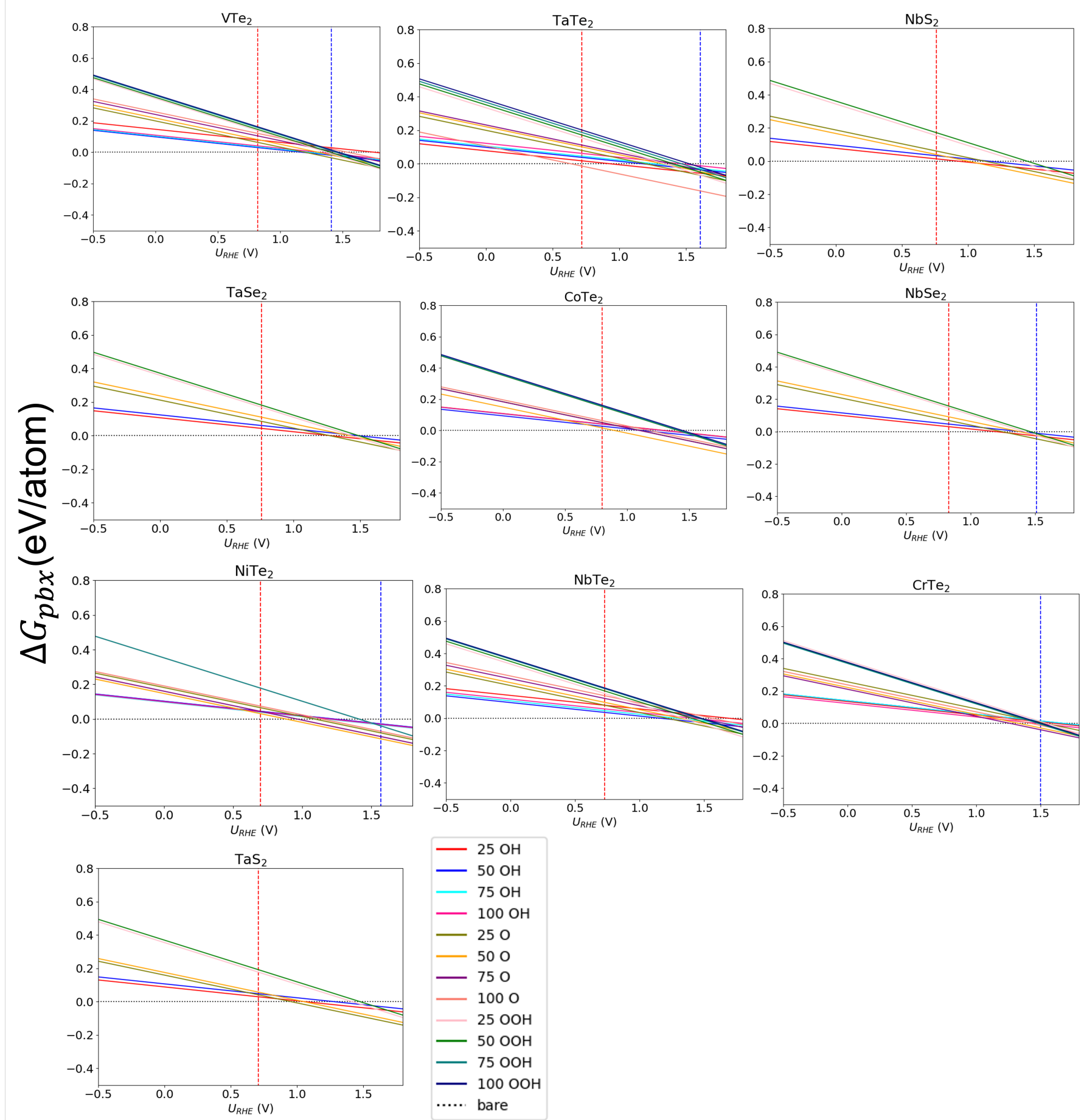

Figure S7: Surface Pourbaix plots for the active materials with H-phase. The red and blue line denote the limiting potential ranges as obtained for ORR and OER respectively considering $25 \%$ surface coverage of the intermediates with the grand canonical potential simulation scheme. 

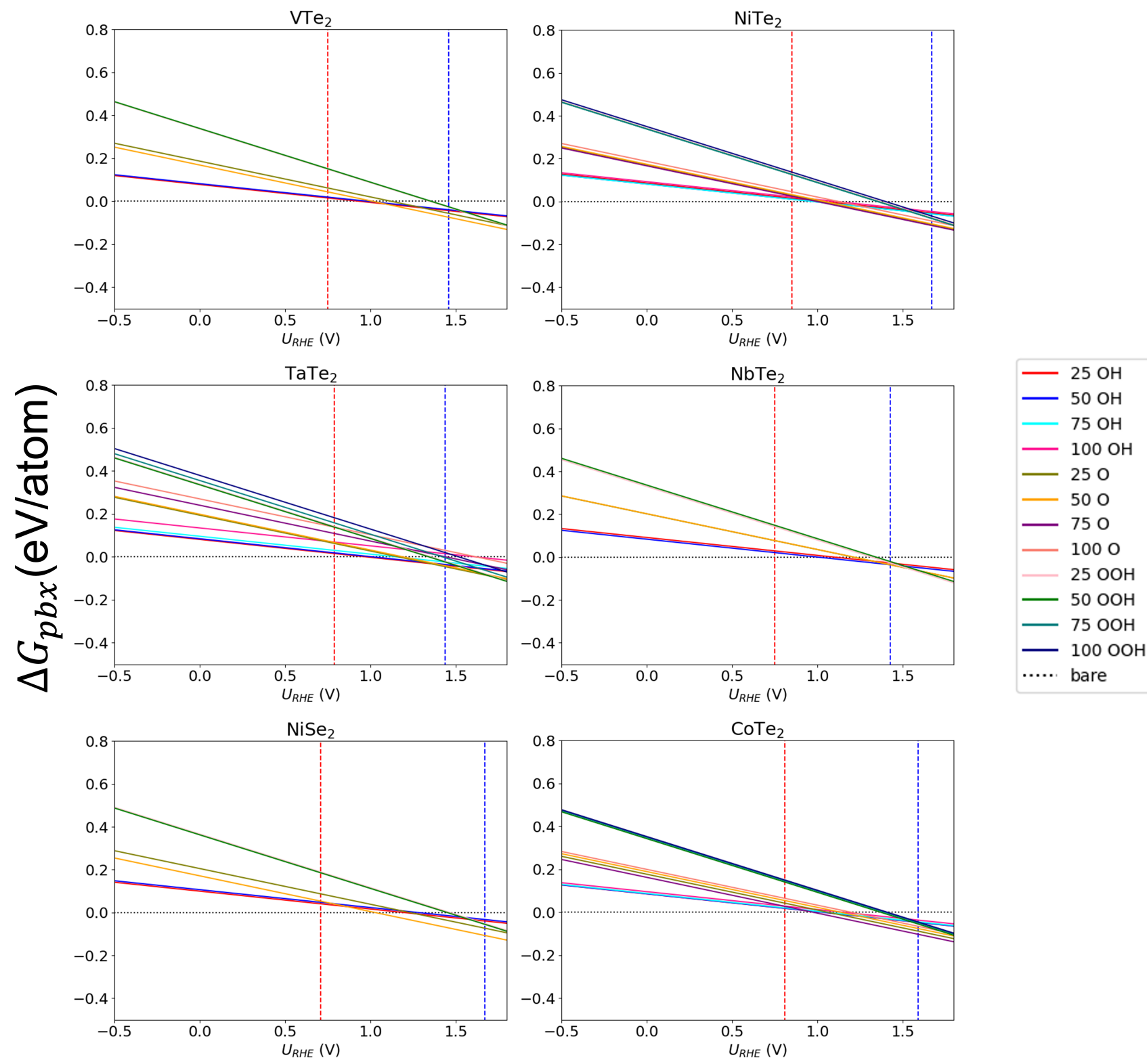

Figure S8: Surface Pourbaix plots for the active materials with T-phase.The red and blue region line the limiting potential ranges as obtained for ORR and OER respectively considering $25 \%$ surface coverage of the intermediates with the grand canonical potential simulation scheme. 

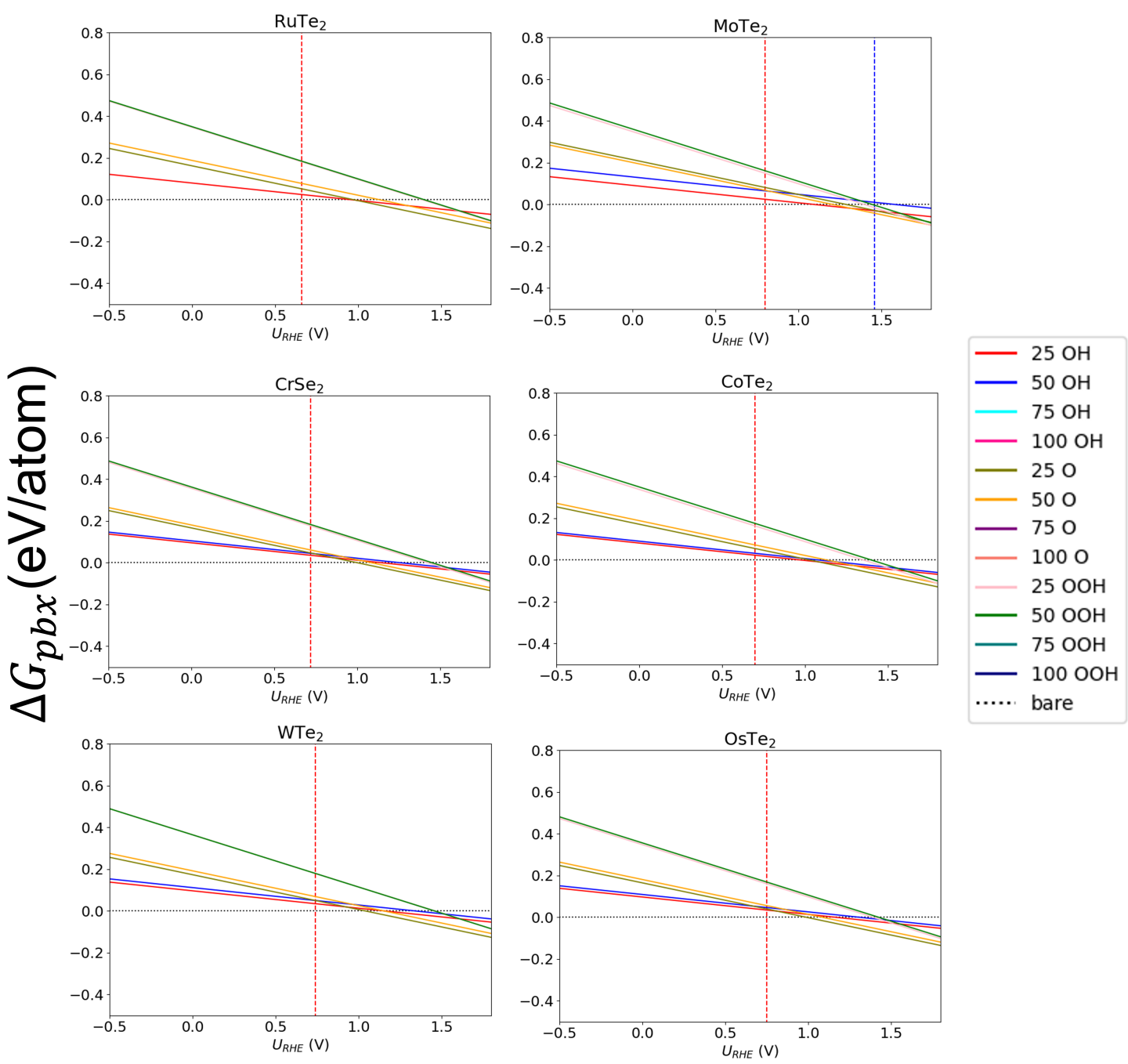

Figure S9: Surface Pourbaix plots for the active materials with T'-phase. The red and blue line denote the limiting potential ranges as obtained for ORR and OER respectively considering $25 \%$ surface coverage of the intermediates with the grand canonical potential simulation scheme. 


\section{References}

(1) Giannozzi, P. et al. QUANTUM ESPRESSO: a modular and open-source software project for quantum simulations of materials. J. Phys.: Condens. Matter 2009, 21, 395502.

(2) Lee, K.; Murray, É. D.; Kong, L.; Lundqvist, B. I.; Langreth, D. C. Higher-accuracy van der Waals density functional. Phys. Rev. B 2010, 82, 081101.

(3) Cooper, V. R. Van der Waals density functional: An appropriate exchange functional. Phys. Rev. B 2010, 81, 161104.

(4) Hamada, I.; Otani, M. Comparative van der Waals density-functional study of graphene on metal surfaces. Phys. Rev. B 2010, 82, 153412.

(5) Prandini, G.; Marrazzo, A.; Castelli, I. E.; Mounet, N.; Marzari, N. Precision and efficiency in solid-state pseudopotential calculations. npj Comput. Mater. 2018, 4, 72.

(6) Lejaeghere, K. et al. Reproducibility in density functional theory calculations of solids. Science 2016, 351 .

(7) Marzari, N.; Vanderbilt, D.; De Vita, A.; Payne, M. C. Thermal Contraction and Disordering of the Al(110) Surface. Phys. Rev. Lett. 1999, 82, 3296-3299.

(8) Andreussi, O; Marzari, N. Electrostatics of solvated systems in periodic boundary conditions. Phys. Rev. B 2014, 90, 245101.

(9) Nattino, F.; Truscott, M.; Marzari, N.; Andreussi, O. Continuum models of the electrochemical diffuse layer in electronic-structure calculations. J. Chem. Phys. 2019, 150, 041722.

(10) Andreussi, O.; Dabo, I.; Marzari, N. Revised self-consistent continuum solvation in electronic-structure calculations. J. Chem. Phys. 2012, 136, 064102. 
(11) Dupont, C.; Andreussi, O.; Marzari, N. Self-consistent continuum solvation (SCCS): The case of charged systems. J. Chem. Phys. 2013, 139, 214110.

(12) Andreussi, O.; Nattino, F.; Dabo, I.; Timrov, I.; Fisicaro, G.; Goedecker, S.; Marzari, N. Environ 1.1: a library for environment effect in first-principles simulations of materials. Environ_1.0 2018, www.quantum $\backslash$-environ.org.

(13) Giannozzi, P., et al. Advanced capabilities for materials modelling with Quantum ESPRESSO. J. Phys.: Condens. Matter 2017, 29, 465901.

(14) Fisicaro, G.; Genovese, L.; Andreussi, O.; Marzari, N.; Goedecker, S. A generalized Poisson and Poisson-Boltzmann solver for electrostatic environments. J. Chem. Phys. 2016, $144,014103$.

(15) Hörmann, N. G.; Andreussi, O.; Marzari, N. Grand canonical simulations of electrochemical interfaces in implicit solvation models. J. Chem. Phys. 2019, 150, 041730.

(16) Karmodak, N.; Andreussi, O. Catalytic Activity and Stability of Two-Dimensional Materials for the Hydrogen Evolution Reaction. ACS Energy Lett. 2020, 5, 885-891.

(17) Mills, G.; Jónsson, H. Quantum and thermal effects in H 2 dissociative adsorption: Evaluation of free energy barriers in multidimensional quantum systems. Phys. Rev. Lett. 1994, 72, 1124.

(18) Henkelman, G.; Jónsson, H. Improved tangent estimate in the nudged elastic band method for finding minimum energy paths and saddle points. J. Chem. Phys. 2000, 113, 9978-9985.

(19) Haastrup, S.; Strange, M.; Pandey, M.; Deilmann, T.; Schmidt, P. S.; Hinsche, N. F.; Gjerding, M. N.; Torelli, D.; Larsen, P. M.; Riis-Jensen, A. C., et al. The Computational 2D Materials Database: high-throughput modeling and discovery of atomically thin crystals. 2D Mater. 2018, 5, 042002. 
(20) Mounet, N.; Gibertini, M.; Schwaller, P.; Campi, D.; Merkys, A.; Marrazzo, A.; Sohier, T.; Castelli, I. E.; Cepellotti, A.; Pizzi, G., et al. Two-dimensional materials from high-throughput computational exfoliation of experimentally known compounds. Nat. Nanotechnol. 2018, 13, 246-252.

(21) Tagawa, M.; Yokota, K.; Ohmae, N.; Matsumoto, K.; Suzuki, M. Hyperthermal Atomic Oxygen Interaction with MoS 2 Lubricants Relevance to Space Environmental Effects in Low Earth Orbit-Atomic Oxygen-Induced Oxidation. Tribol. Lett. 2004, 17, 859-865.

(22) Walter, T. N.; Kwok, F.; Simchi, H.; Aldosari, H. M.; Mohney, S. E. Oxidation and oxidative vapor-phase etching of few-layer MoS2. J. Vac. Sci. Technol., B: Nanotechnol. Microelectron.: Mater., Process. 2017, 35, 021203.

(23) Farigliano, L. M.; Paredes-Olivera, P. A.; Patrito, E. M. Initial Steps of Oxidative Etching of MoS2 Basal Plane Induced by O2. J. Phys. Chem. C 2020, 124, 1317713186. 